\title{
MAGNETIC FIELD CORRECTION IN NUCLOTRON
}

\author{
I. B. Issinsky, V. A. Mikhailov, V. A. Shchepunov \\ Laboratory of High Energies \\ Joint Institute for Nuclear Research, Dubna, Russia 141980
}

\begin{abstract}
The descripton of the Nuclotron magnetic field correction is presented. The influence of the structure magnet misalignments and the field imperfections obtained from magnet measurements on the beam dynamical parameters is considered, and the correction schemes are rewiewed. This influence and the correction efficiency are investigated with numerical methods.
\end{abstract}

\section{INTRODUCTION}

To provide long-term prospects in the area of both relativistic nuclear and applied physics a project of accelerator facilities named Nuclotron has been developed in the Laboratory of High Energies JINR [1]. This project includes a superconducting synchrotron (under commissioning now) and a booster synchrotron [2]. The existing linac used as an injector can accelerate ions from deuterium to xenon with charge to mass ratio $\mathrm{Z} / \mathrm{A}$ in the range $0.33-0.50$. In the Nuclotron ions are accelerated up to relativistic energies of $6 \mathrm{GeV} / \mathrm{u}$. General parameters of the $\mathrm{Nu}$ clotron ring [3] are given in the table 1. The lattice of Nuclotron includes 8 superperiods. The structure of one superperiod and its optical functions are shown in fig. 1. Each superperiod contains 3 regular FODO cells with dipole magnets and 1 cell with large drift spaces occupied with elements of the injection-extraction systems, RF-voltages, the internal target equipment etc. Small drift spaces at the lattice quadrupoles are used for the diagnostic elements and multipolar magnets of the magnetic field correction systems.

The correction system includes 20 pick-up electrodes, tune and intensity measurement systems, 29 superconducting multipolar correcting magnets correcting closed orbit (C.O.) [5], chromaticity and octupolar detuning [6], betatron resonances up to the 4-th order [9]. Nuclotron operates with the horizontal and vertical tunes $\left(\mathrm{Q}_{x}\right.$ and $\left.\mathrm{Q}_{z}\right)$ between 6 and 7 with the nominal operating point at $\mathrm{Q}_{x}=6.80, \mathrm{Q}_{z}=6.85$ (see fig.2).

\section{MULTIPOLAR CORRECTOR}

Multipolar corrector [4] has 4 superconducting windings. Two of them, skew and right dipoles, are used for the C.O. correction. Other windings are of quadrupolar, sextupolar or octupolar types (skew or regular). All windings are placed on a cylindrical framework wrapped with the hollow tube. Helium flow inside the tube cools the windings due to a thermal contact. The iron cylindrical yoke around the windings allows to encrease the maximal field in the center of a corrector by a factor of 1.7-1.8. The yoke and windings are placed in a cryostat cooled with liquid nitrogen.

\section{CLOSED ORBIT CORRECTION}

Table 2 presents calculated and measured maximal values of C.O. distortions. It can be seen that at injection $\left(\mathrm{B}_{i n j}=291\right.$

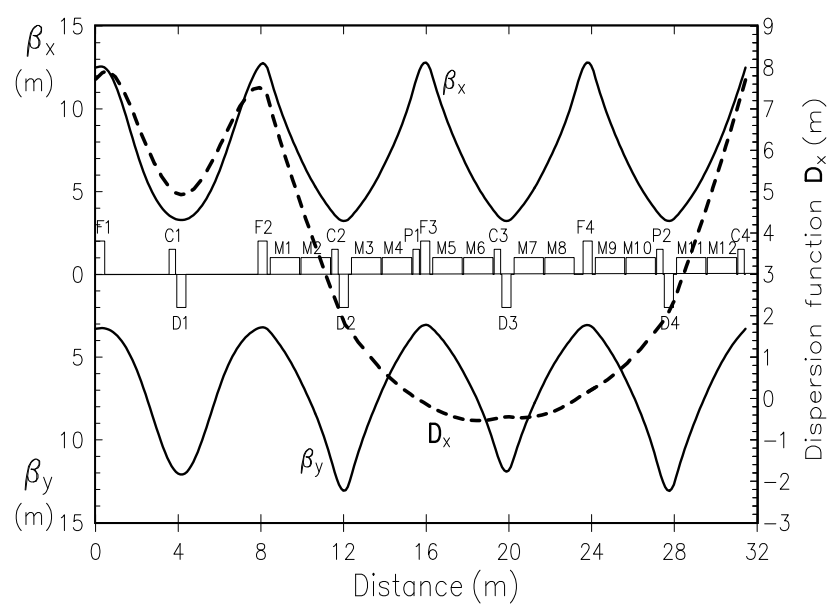

Figure. 1. Twiss $\beta_{x, z}$ and dispersion $D_{x}$ functions of the $\mathrm{Nu}-$ clotron superperiod. F, D - focusing and defocusing quadrupoles respectively; $\mathrm{M}$ - dipole magnets; $\mathrm{C}$ - multipolar correctors; $\mathrm{P}$ pick-ups. The positions of the correctors and pick-ups are shown for the 1-st superperiod.

Gauss) remanent fields give the main contribution to the vertical orbit distortions, while the horizontal distortions are mainly determined by the dispersion of integrated fields in dipole magnets. Four conventional C.O. correction algorithms are used for Nuclotron: least squares, harmonical, bump correction and MICADO method. It was found in numerical simulation studies [5] that $3.5 \mathrm{~mm}$ maximal orbit distortions could be reached with corrections in both planes.

\section{TUNE, CHROMATICITY AND AMPLITUDE DETUNING CORRECTIONS}

Dipoles, F and D quadrupoles of the lattice have 3 independent power supplies. To keep tunes constant for the cycle of acceleration the ratios $\mathrm{I}_{F, D} / \mathrm{I}_{D i p}$ of the currents $\mathrm{I}_{F, D}$ in $\mathrm{F}$ and $\mathrm{D}$ quadrupoles to the current $\mathrm{I}_{\text {Dip }}$ in dipoles are changed to compensate for the changing the values $(\mathrm{BL})_{F, D} /(\mathrm{BL})_{D i p}$, where (BL)-average integrated fields in quadrupoles and dipoles (that is mainly due to the saturation phenomena). At constant $\mathrm{I}_{F, D} / \mathrm{I}_{D i p}$, the tune shifts can be as large as +0.45 in the range of the main field $B_{o}=1.7-2.0 \mathrm{~T}$.

For the chromaticity $\left(\chi_{x}=-7.7, \chi_{z}=-7.9\right)$ correction there are 2 families of sextupolar windings placed in the multipolar correctors of each superperiod. The same correctors also contain 2 families of octupolar windings to compensate for the amplitude detunings. The currents of the families are chosen so that the tunes of particles move away from the line of the resonance $Q_{x}-Q_{z}=0$ with the increase of the amplitudes of their oscillations. It is also possible to use complicated sextupolar-octupolar 


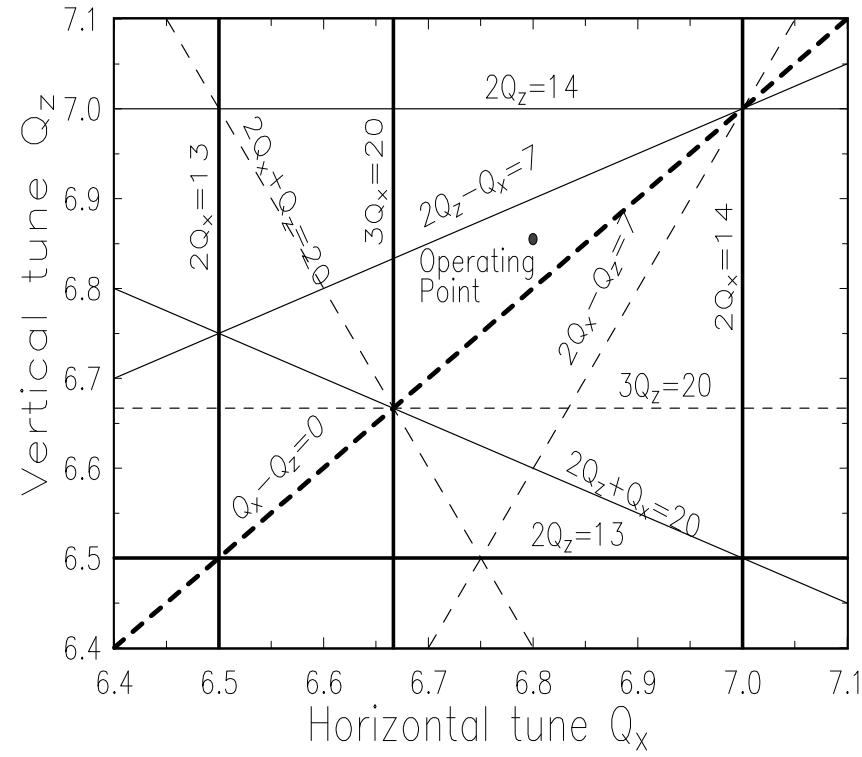

Figure. 2. 2-nd and 3-d order resonances and Nuclotron operating point (O.P.).

correction schemes that take into account the 2-nd order sextupolar effects [6].

\section{CORRECTION OF BETATRON RESONANCES}

Table 3 gives the list of resonances up to the 4-th order to be corrected (see the diagram in fig.2) and their r.m.s. stopbands estimated with the magnet measurements [7] and geodesic data. The correction system is capable of correcting resonances 1-2 and 3-4 simultaneously, i.e. it can correct each of these resonances without affecting the correction of the others. For the 3-d and the 4-th order resonances such simultaneous corrections are possible only for the combinations of sum and difference ones: $5-6$ or $5-7 ; 8-9$ or $8-10 ; 11-12$ or $11-13 ; 14-15$ or $14-16$. Generally, correctors of the resonance $m Q_{x}+n Q_{z}=\mathrm{N}$ create the $\mathrm{N}$-th appropriate multipole harmonic. The sinus $\mathrm{S}$ and cosinus $\mathrm{C}$ components of this harmonic have the following dependence on the main filed Bo:

$$
\mathrm{C}=\mathrm{C}_{r}+C_{b} B_{o}+C_{t}\left(d B_{o} / \mathrm{dt}\right), \quad \mathrm{S}=\mathrm{S}_{r}+S_{b} B_{o}+S_{t}\left(d B_{o} / \mathrm{dt}\right),
$$

where the constant terms $\mathrm{C}_{r}$ and $\mathrm{S}_{r}$ are due to remanent fields, while those proportional $\mathrm{B}_{o}$ and the derivative $\mathrm{dB}_{o} / \mathrm{dt}$ are presumably due to misalignments and/or field imperfections, and eddy currents respectively. It is the terms proportional to $\mathrm{B}_{o}$ that were taken into account in the estimations of the stopbands given in the table 2 . The eddy current terms have been estimated according to [8]. For the Nuclotron vacuum chamber width $\mathrm{a}=0.5$ $\mathrm{mm}$ and $\left(\mathrm{dB}_{o} / \mathrm{dt}\right)_{\max }=20 \mathrm{kG} / \mathrm{s}$ they were found to be quite small in comparison with the others. The remanent field contribution is considerable and should be carefully taken into account. Full experimental data on the betatron resonance corrections will be obtained in the planned Nuclotron runs. The detailed description of the resonance correction schemes and the possible sources of relevant magnetic imperfections are discussed in [9].
Table I

General Nuclotron parameters

\begin{tabular}{|l|c|}
\hline Injection energy for nuclei & $5 \mathrm{MeV} / \mathrm{u}$ \\
for protons & $20 \mathrm{MeV}$ \\
Max energy for nuclei $(\mathrm{q} / \mathrm{A}=0.5)$ & $6 \mathrm{GeV} / \mathrm{u}$ \\
for protons & $12.8 \mathrm{GeV}$ \\
Circumference & $251.52 \mathrm{~m}$ \\
Duration of acceleration & $(0.5-1.5) \mathrm{sec}$ \\
Max accelerating voltage & $50 \mathrm{kV}$ \\
Transition energy & $8.6 \mathrm{GeV}$ \\
Field in dipoles at injection & $0.029 \mathrm{~T}$ \\
& $2.083 \mathrm{~T}$ \\
Gradient in quads at injection & $0.490 \mathrm{~T} / \mathrm{m}$ \\
maximum & $34.6 \mathrm{~T} / \mathrm{m}$ \\
Betatron tunes $\mathrm{Q}_{x, z}$ & $6.8,6.85$ \\
Chromaticity $\chi_{x, z}=\delta Q_{x, z} / \delta \mathrm{p} / \mathrm{p}$ & $-7.7,-7.9$ \\
Compaction factor & 0.0135 \\
Max closed orbit $($ after correction) & $3.5 \mathrm{~mm}$ \\
Acceptance horizontal & $40 \pi \mathrm{mm} \mathrm{mrad}$ \\
vertical & $45 \pi \mathrm{mm} \mathrm{mrad}$ \\
Emittance at injection & $30 \pi \mathrm{mm} \mathrm{mrad}$ \\
minimum & $2 \pi \mathrm{mm} \mathrm{mrad}$ \\
Maximum momentum spread & $\mp 4 \times 10^{-3}$ \\
\hline
\end{tabular}

Table II

Calculated values of maximal closed orbit distortions [mm] due to different sources of imperfections and measured distortions.

$$
\mathrm{B}_{o}=471 \text { Gauss. }
$$

\begin{tabular}{|l|c|c|}
\hline $\begin{array}{l}\text { Source of } \\
\text { imperfection }\end{array}$ & $\begin{array}{c}\text { Vertical } \\
\text { distortion }\end{array}$ & $\begin{array}{c}\text { Horizontal } \\
\text { distortion }\end{array}$ \\
\hline Lense misalignments & 1.3 & 2.0 \\
Dipole tilts & 0.6 & 0.0 \\
(BL) dispersion in dipoles & 0.0 & 11.0 \\
Season ground motion & 2.1 & 3.6 \\
Remanent fields & 18.2 & 4.2 \\
\hline Measured values & 18 & 20 \\
\hline
\end{tabular}

\section{ALGORITHMS FOR THE NUMERICAL INVESTIGATION OF THE CORRECTION EFFICIENCY}

The correction efficiencies have been investigated numerically with Monte-Carlo simulation algorithms. Generally, one step of the simulation includes: 1) random generation of multipolar field components or magnet misalignments according to their measured average and r.m.s. values; 2) calculation of the maximum values of C.O. distortions, or tracking test particles to determine tune spreads and/or increase of effective emittance; 3 ) calculation of corrector strengths according to a correction algorithm; 4) repetition of the step 2 taking into account fields in correctors. At the end of the cycle the statistical distributions of the maximal C.O. distortions, tune spreads or effective emittances before and after correction are plotted.

Figure 3 gives an example of the symultaneous correction of the resonances $2 \mathrm{Q}_{x}=13$ and $2 \mathrm{Q}_{z}=13$. The resonances are driven 
Table III

Corrected betatron resonances and estimated r.m.s. stopbands $\langle\mathrm{d}\rangle$ at $\mathrm{Q}_{x}=6.80, \mathrm{Q}_{z}=6.85$, emittances $\mathrm{E}_{x, z}=30 \pi \mathrm{mm} \mathrm{mrad}$ and $\mathrm{B}_{o}=2.33 \mathrm{kG}$.

\begin{tabular}{|l|l|c|c|c|}
\hline $\mathrm{N}$ & Resonance & $100\langle\mathrm{~d}\rangle$ & $\begin{array}{c}\text { Type of } \\
\text { field }\end{array}$ & $\begin{array}{c}\text { Number of } \\
\text { correctors }\end{array}$ \\
\hline 1 & $2 \mathrm{Q}_{x}=13,14$ & 10.5 & regular & 8 \\
2 & $2 \mathrm{Q}_{z}=13,14$ & 10.5 & quad. & \\
\hline 3 & $Q_{x}-Q_{z}=0$ & 6.0 & skew & 4 \\
4 & $\mathrm{Q}_{x}+Q_{z}=13$ & 6.5 & quad. & \\
\hline 5 & $2 \mathrm{Q}_{z}-Q_{x}=7$ & 0.55 & regular & \\
6 & $2 \mathrm{Q}_{z}+Q_{x}=20$ & 0.60 & sext. & 4 \\
7 & $3 \mathrm{Q}_{x}=20$ & 0.30 & & \\
\hline 8 & $2 \mathrm{Q}_{x}-Q_{z}=7$ & 0.35 & skew & \\
9 & $2 \mathrm{Q}_{x}+Q_{z}=20$ & 0.41 & sext. & 4 \\
10 & $3 \mathrm{Q}_{z}=20$ & 0.20 & & \\
\hline 11 & $2 \mathrm{Q}_{x}-2 Q_{z}=0$ & 0.10 & regular & \\
12 & $4 \mathrm{Q}_{x, z}=27$ & 0.03 & oct. & 4 \\
13 & $2 \mathrm{Q}_{x}+2 Q_{z}=27$ & 0.08 & & \\
\hline 14 & $3 \mathrm{Q}_{z}-Q_{x}=13$ & 0.09 & skew & \\
15 & $3 \mathrm{Q}_{x}+Q_{z}=27$ & 0.11 & oct. & 4 \\
16 & $3 \mathrm{Q}_{z}+Q_{x}=27$ & 0.11 & & \\
\hline
\end{tabular}

by the dispersion $\langle\mathrm{dL} / \mathrm{L}\rangle$ of the efective lengths of the structure quadrupoles at the fields of injection. $\mathrm{I}_{x}$ denotes the linear invariant $\left[x^{2}+\left(x^{\prime} b+x \alpha\right)^{2}\right] / 2 \beta_{x}$ in the horizontal plane, and $\mathrm{I}_{z}-$ in the vertical. To determine the values of $(\delta I / I)_{x, z}^{\max } 4$ test particles were tracked for 20 revolutions for each of the 100 random sets of quadrupolar magnets.

\section{ACKNOWLEDGMENTS}

The authors would like to thank A. M. Donyagin and M. A. Voyevodin for magnetic measurements data, A. P. Tsarenkov for the closed orbit measurements, O. S. Kozlov and A. P. Tsarenkov for fruitful discussions of the problem.

\section{References}

[1] Baldin A. M. et al., Nuclotron Status Report, IEEE Trans. on Nucl. Sci., NS-30, 4:3247 (1983).

[2] Issinsky I. B., Mikhailov V. A., Shchepunov V. A., Nuclotron Lattice, In: Proc. of the 2-nd EPAC, Nice, 1990, Vol.1, 458.

[3] Issinsky I. B., Mikhailov V. A., Conception of the 200 $\mathrm{MeV} / \mathrm{u}$ booster for the Nuclotron, In: Proc. of IEEE PAC, San-Francisco, California, May 6-9 1991, Vol.5, 2886.

[4] Anishchenko N. G. et al., The Model of the Superconducting Multipolar Corrector for Nuclotron, In: Proc. of XI AllUnion Conf. on Particle Accelerators, vol.II, Dubna, 1989, p.221.

[5] Mikhailov V. A., Shchepunov V. A., Magnetic Field Correction System of Nuclotron, In.: Proc. of XIII Russian Conf. on Particle Accelerators, Dubna, 1992, p.253.

[6] Shchepunov V. A., Betatron Tune Spread Corrections in Nuclotron, JINR, P9-92-203, Dubna, 1992.
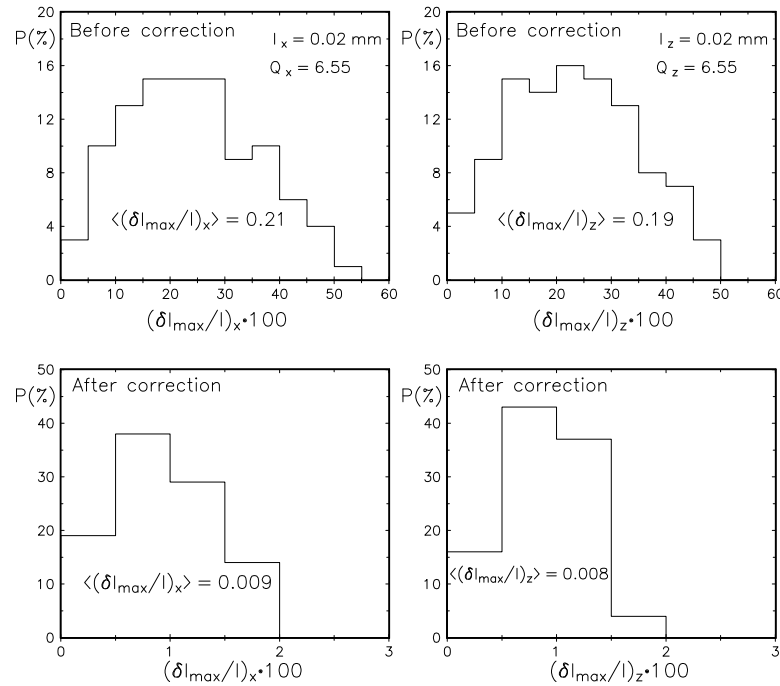

Figure. 3. Statistical distributions for maximal distortions $(\delta I / I)_{x, z}^{\max }$ of linear invariants $\mathrm{I}_{x, z}$ under the influence of resonances $2 \mathrm{Q}_{x, z}=13$ before and after correction with 4 correctors. The simulation was performed for 100 random sets of effective lengths of the lattice quadrupoles with dispersion $\langle\mathrm{dL} / \mathrm{L}\rangle$ $=4.5 \times 10^{-3}$.

[7] Donyagin A. M., Eliseeva I. A., Kovalenko A. D., Analysis and Corrections of the Magnetic Field Imperfections of the Nuclotron SC Dipole Magnets, JINR, P9-88-644, Dubna, 1988.

[8] Orlov Yu. F. and Heifets S. A., The Distortion of the Magnetic Field of a Strong Focusing Accelerator Due to a Metal Vacuum Chamber, PTE, N1, 1959, p.21.

[9] Mikhailov V. A., Shchepunov V. A., Correction of Betatron Resonances in Nuclotron, JINR, P9-92-204, Dubna, 1992. 